\title{
111. Einige Darstellungen analytischer Funktionen und ihre Anwendungen auf konforme Abbildung.
}

Von Yûsaku Komatu.

Mathematisches Institut, Kaiserliche Universität zu Tokyo.

(Comm. by S. KAKEYA, M.I.A., Oct. 12, 1944.)

1. Herglotzsche Darstellung. Wir betrachten zuerst die Familie aerjenigen im Grundgebiet $|z|<1$ regulär analytischen Funktionen $\{\emptyset(z)\}$, für welche die Größe

$$
\rho(r, \varphi)=\int_{0}^{\varphi} \Re \varphi\left(r e^{i \theta}\right) d \theta \quad(0 \leqq \varphi \leqq 2 \pi, 0 \leqq r<1)
$$

als Funktion von $\varphi$ für alle $r<1$ gleichmäßig beschränkt und von beschränkter Schwankung ist. Dann läßt sich nach einem Hellyschen Satz eine monoton wachsende Zahlenfolge $\left\{r_{n}\right\}, r_{n} \rightarrow 1(n \rightarrow \infty)$, so auswählen, daß die Limesfunktion

$$
\rho(\varphi)=\lim _{n \rightarrow \infty} \rho\left(r_{n}, \varphi\right)
$$

höchstens bis auf eine Nullmenge von $\varphi$ existiert und selbst beschränkt und von beschränkter Schwankung ist. Somit ergibt sich bekanntlich die Poisson-Stieltjessche Darstellung nach Herglotz

$$
\Phi(z)=\frac{1}{2 \pi} \int_{0}^{2 \pi} \frac{e^{i \varphi}+z}{e^{i \varphi}-z} d \rho(\varphi)+i \Im \Phi(0) .
$$

Die genannten Bedingungen für $\rho(r, \varphi)$ sind gewiß dann erfüllt, falls entweder $\Re \varphi\left(r e^{i \varphi}\right)$ einerseits oder $\int_{0}^{2 \pi}\left|\Re \varphi\left(r e^{i \theta}\right)\right| d \theta$ beschränkt bleibt.

2. Darstellung für $z f^{\prime \prime}(z) \mid f^{\prime}(z)$ und verwandte Größen. Wir nehmen nun an, daß eine Funktion $f(z)$ im Einheitskreis regulär analytisch und im abgeschlossenen Einheitskreis stückweise regulär sei. Sie verhält also in $|z| \leqq 1$ abgesehen von gewissen endlich vielen Randpunkten

$$
z=e^{i \varphi_{\mu}} \quad(\mu=1, \ldots, m), \quad 0<\varphi_{1}<\cdots<\varphi_{m} \leqq 2 \pi,
$$

regulär und besitzt in jedem solchen Punkte beide bestimmte Grenzwerte $\lim f^{\prime}\left(e^{i \varphi}\right)$ für $\varphi \rightarrow \varphi_{\mu}-0$ sowie für $\varphi \rightarrow \varphi_{\mu}+0$. Ferner setzen wir jetzt voraus, da $B$ ihre Ableitung $f^{\prime}(z)$ überall in $|z| \leqq 1$ höchstens bis auf solche Ausnahmerandpunkte nullstellenfrei sei. Bezeichnen wir die Sprunghöhe längs der Peripherie von $\arg f^{\prime}\left(e^{i \varphi}\right)$ im Punkte $e^{i \varphi_{\mu}}$ mit

so ist die Funktion

$$
\left(1-\alpha_{\mu}\right) \pi=\arg \frac{f^{\prime}\left(e^{i\left(\varphi_{\mu}+0\right)}\right)}{f^{\prime}\left(e^{i\left(\varphi_{\mu}-0\right)}\right)}
$$




$$
\begin{aligned}
\Phi(z) & =z \frac{d}{d z} \lg \left[f ^ { \prime } ( z ) \prod _ { n = 1 } ^ { m } \left(z-e^{\left.\left.i \varphi_{\mu}\right)^{1-a_{\mu}}\right]}\right.\right. \\
& =\frac{z f^{\prime \prime}(z)}{f^{\prime}(z)}+\sum_{\mu=1}^{n} \frac{\left(1-\alpha_{\mu}\right) z}{z-e^{i \varphi_{\mu}}}
\end{aligned}
$$

regulär analytisch im ganzen $|z| \leqq 1$, und überdies verschwindet sie in $z=0$. Wendet man die obengenannte Herglotzsche Darstellung auf diese Funktion, so ergibt sich unmittelbar die Gleichung

$$
\frac{z f^{\prime \prime}(z)}{f^{\prime}(z)}=\frac{1}{2 \pi} \int_{0}^{2 \pi} \frac{e^{i \varphi}+z}{e^{i \varphi}-z} d \rho(\varphi)-\sum_{\mu=1}^{m} \frac{\left(1-\alpha_{\mu}\right) z}{z-e^{i \varphi}} ;
$$

wegen der Regularität von $\Phi(z)$ im ganzen $|z| \leq 1$ ist hierbei

$$
\begin{aligned}
\rho(\varphi) & =\int_{0}^{\varphi} \Re \Phi\left(e^{i \theta}\right) d \theta=\lim _{r \rightarrow 1}\left\{\Re \int_{0}^{\varphi} \frac{r e^{i \theta} f^{\prime \prime}\left(r e^{i \theta}\right)}{f^{\prime}\left(r e^{i \theta}\right)} d \theta+\sum_{\mu=1}^{m}\left(1-\alpha_{\mu}\right) \Re \int_{0}^{\varphi} \frac{r e^{i \theta}}{r e^{i \theta}-e^{i \varphi}} d \theta\right\} \\
& =\arg \frac{f^{\prime}\left(e^{i \varphi}\right)}{f^{\prime}(1)}+\sum_{\mu=1}^{m}\left(1-\alpha_{\mu}\right)\left(\frac{\varphi}{2}-\pi \varepsilon_{\mu}(\varphi)\right)
\end{aligned}
$$

worin zur Akürzung mit $\varepsilon_{\mu}(\varphi)$ diejenige Treppenfunktion bezeichnet ist, welche gleich 0 oder 1 ist je nach $0 \leqq \varphi<\varphi_{\mu}$ oder $\varphi_{\mu}<\varphi \leqq 2 \pi$. Wegen der sofort feststellbaren Beziehungen

$$
\frac{1}{2 \pi} \int_{0}^{2 \pi} \frac{e^{i \varphi}+z}{e^{i \varphi}-z} d\left(\frac{\varphi}{2}-\pi \varepsilon_{\mu}(\varphi)\right)=\frac{1}{2}-\frac{1}{2} \frac{e^{i \varphi_{\mu}}+z}{e^{i \varphi_{\mu}-z}}=\frac{z}{z-e^{i \varphi_{\mu}}} \quad(\mu=1, \ldots, m)
$$

ergibt sich also die Darstellung

$$
\frac{z f^{\prime \prime}(z)}{f^{\prime}(z)}=\frac{1}{2 \pi} \int_{0}^{2 \pi} \frac{e^{i \varphi}+z}{e^{i \varphi}-z} d \arg f^{\prime}\left(e^{i \varphi}\right),
$$

und indem man die durch Einsetzen von $z=0$ aus ihr entstehende Relation von ihr selbst subtrahiert, läßt sie sich auch in die Form

$$
\frac{f^{\prime \prime}(z)}{f^{\prime}(z)}=\frac{1}{\pi} \int_{0}^{2 \pi} \frac{1}{e^{i \varphi}-z} d \arg f^{\prime}\left(e^{i \varphi}\right)
$$

bringen, und da offenbar

$$
\int_{0}^{2 \pi} \frac{1}{e^{i \varphi}-z} d \varphi=0, \quad \arg \frac{d f\left(e^{i \varphi}\right)}{d \varphi}=\frac{\pi}{2}+\varphi+\arg f^{\prime}\left(e^{i \varphi}\right)
$$

sind, gilt weiter die Darstellung der Gestalt

$$
\frac{f^{\prime \prime}(z)}{f^{\prime}(z)}=\frac{1}{\pi} \int_{0}^{2 \pi} \frac{1}{e^{i \varphi}-z} d \arg d f\left(e^{i \varphi}\right)
$$

Integration über $z$ zieht also nach sich

$$
\lg \frac{f^{\prime}(z)}{f^{\prime}(0)}=\frac{1}{\pi} \int_{0}^{2 \pi} \lg \frac{e^{i \varphi}}{e^{i \varphi}-z} d \arg f^{\prime}\left(e^{i \varphi}\right)=\frac{1}{\pi} \int_{0}^{2 \pi} \lg \frac{e^{i \varphi}}{e^{i \varphi}-z} d \arg d f\left(e^{i \varphi}\right)
$$

und insbesondere folgt weiter daraus nach Trennen des reellen Teils die Beziehung 


$$
\lg \left|\frac{f^{\prime}\left(r e^{i \theta}\right)}{f^{\prime}(0)}\right|=-\frac{1}{\pi} \int_{0}^{2 \pi} \lg \sqrt{(1-r)^{2}+4 r \sin ^{2} \frac{\theta-\varphi}{2}} d \arg f^{\prime}\left(e^{i \varphi}\right)
$$

Läßt man hier $r$ gegen 1 streben, so erhält man die Beziehung

$$
\lg \left|\frac{f^{\prime}\left(e^{i \theta}\right)}{f^{\prime}(0)}\right|=-\frac{1}{\pi} \int_{0}^{2 \pi} \lg 2\left|\sin \frac{\theta-\varphi}{2}\right| d \arg f^{\prime}\left(e^{i \varphi}\right),
$$

welche für eine Gleichung in bezug auf die Randwerte von $f^{\prime}(z)$ angesehen werden kann. Wir bemerken nebenbei, daß die letzte Gleichung auf ein Problem über die ebene Potentialströmung um ein Tragflügelprofil anwendbar ist, was ich in einer demnächst in dem Journ. Soc. Aeronaut. Sci. Nippon erscheinenden Note zeigen will.

Wir betrachten nun eine analytische Funktion $f(z)$, und nehmen hierbei zunächst an, daß das Integral $\int_{0}^{z} f(z) d z$ den Bedingungen über Regularität für die in den obigen Úberlegungen betrachteten Funktionen $f(z)$ genüge. Hier wollen wir aber etwas allgemeiner zulassen, da $B$ die Funktion $f(z)$ selbst in $|z|<1$ eine endliche Anzahl der Nullstellen $a_{x}(x=1, \ldots, N)$ und der Pole $b_{\lambda}(\lambda=1, \ldots, P)$ besitzen darf, wobei solche Stellen natürlich ihrer Mehrfachheit yemäß ausgezählt werden sollen. Wenn man demgemäB die Funktion

$$
F(z)=f(z) \prod_{\lambda=1}^{p} \frac{z-b}{1-\bar{b}_{\lambda} z} / \prod_{x=1}^{N} \frac{z-a_{x}}{1-\bar{a}_{x} z}
$$

betrachtet und dann auf die damit gebildete in $|z| \leqq 1$ reguläre Funktion

$$
\begin{aligned}
\Phi(z) & =z \frac{d}{d z} \lg \left[F ( z ) \prod _ { \mu = 1 } ^ { m } \left(z-e^{\left.\left.i \varphi_{\mu}\right)^{1-a_{\mu}}\right]}\right.\right. \\
& =\frac{z f^{\prime}(z)}{f(z)}-\sum_{x=1}^{N} \frac{\left(1-\left|a_{x}\right|^{2}\right) z}{\left(z-a_{x}\right)\left(1-\bar{a}_{x} z\right)}+\sum_{\lambda=1}^{P} \frac{\left(1-\left|b_{\lambda}\right|^{2}\right) z}{\left(z-b_{\lambda}\right)\left(1-\bar{b}_{\lambda} z\right)}+\sum_{\mu=1}^{m} \frac{\left(1-\alpha_{\mu}\right) z}{z-e^{i \varphi_{\mu}}}
\end{aligned}
$$

die Herglotzsche Darstellung anwendet, so erhält man unter Berücksichtigung der für jeden im Einheitskreis gelegenen Punkt $c$ allgemein geltenden Beziehung

$$
\frac{1}{2 \pi} \int_{0}^{2 \pi} \frac{e^{i \varphi}+z}{e^{i \varphi}-z} d \arg \frac{e^{i \varphi}-c}{1-\bar{c} e^{i \varphi}}=\frac{1+\bar{c} z}{1-\bar{c} z},
$$

nach einigen einfachen Umformungen, die Darstellung

$$
\begin{aligned}
\frac{z f^{\prime}(z)}{f(z)}=\frac{1}{2 \pi} \int_{0}^{2 \pi} \frac{e^{i \varphi}+z}{e^{i \varphi}-z} d \arg f\left(e^{i \varphi}\right) & +\sum_{x=1}^{N}\left(\frac{z}{z-a_{x}}-\frac{1}{1-\bar{a}_{x} z}\right) \\
& -\sum_{\lambda=1}^{P}\left(\frac{z}{z-b_{\lambda}}-\frac{1}{1-\bar{b}_{\lambda} z}\right),
\end{aligned}
$$

und diese letzte läßt sich wegen der offenbar bestehenden Beziehung

$$
\frac{1}{2 \pi} \int_{0}^{2 \pi} d \arg f\left(e^{i \varphi}\right)=N-P
$$


auch in die Gestalt

$$
\begin{aligned}
\frac{f^{\prime}(z)}{f(z)}=\frac{1}{\pi} \int_{0}^{2 \pi} \frac{1}{e^{i \varphi}-z} d \arg f\left(e^{i \psi}\right) & +\sum_{x=1}^{N}\left(\frac{1}{z-a_{k}}-\frac{\bar{a}_{x}}{1-\bar{a}_{x} z}\right) \\
& -\sum_{\lambda=1}^{P}\left(\frac{1}{z-b_{\lambda}}-\frac{\bar{b}_{\lambda}}{1-\bar{b}_{\lambda} z}\right)
\end{aligned}
$$

bringen. Nach Integration ergibt sich daraus weiter

$$
\begin{aligned}
\lg \frac{f(z)}{A z^{h}}=\frac{1}{\pi} \int_{0}^{2 \pi} \lg \frac{e^{i \varphi}}{e^{i \varphi}-z} \cdot d \arg f\left(e^{i \varphi}\right) & +\sum_{x=1}^{N} \lg \left(1-\frac{z}{a_{x}}\right)\left(1-\bar{a}_{x} z\right) \\
& -\sum_{\lambda=1}^{P} \lg \left(1-\frac{z}{b_{\lambda}}\right)\left(1-\bar{b}_{\lambda} z\right),
\end{aligned}
$$

wobei eine i. a. komplexe Konstante $A$ und eine ganze rationale Zahl $k$ derart bestimmt werden $\mathrm{muB}$, daß der Grenzwert

$$
\lim _{z \rightarrow 0} \frac{f(z)}{z^{k}}=A
$$

endlich und nicht verschwindend ist; alle hier auftretenden $\mathrm{Zweige}$ der Logarithmen sollen in $z=0$ verschwinden.

3. Verzerrungssätze für einige Klassen der den Einheitskreis schlicht abbildenden Funktionen. Ich möchte nun kurz darauf aufmerksam machen, da $B$ die oben hergeleiteten Darstellungen in sehr einfacher Weise einige bekannten Verzerrungssätze für gewisse Klassen der im Einheitskreis schlichten Funktionen nach sich ziehen. Betrachten wir etwa eine beliebige in $|z|<1$ normierte reguläre schlichte Funktion

$$
w=f(z) \quad f(0)=0, f^{\prime}(0)=1,
$$

welche $|z|<1$ auf ein Konvexgebiet abbildet, dann gilt bekanntlich für solche Funktion stets die Ungleichung

$$
1+\Re \frac{z f^{\prime \prime}(z)}{f^{\prime}(z)}>0
$$

in $|z|<1$ und folglich wächst der aus ihr gebildete Ausdruck

$$
\rho(r, \varphi)=\int_{0}^{\varphi}\left(1+\Re \frac{r e^{i \theta} f^{\prime \prime}\left(r e^{i \theta}\right)}{f^{\prime}\left(r e^{i \theta}\right)}\right) d \theta=\varphi+\arg \frac{f^{\prime}\left(r e^{i \varphi}\right)}{f^{\prime}(r)}
$$

für jeden Wert von $r(<1)$ monoton in bezug auf $\varphi$. Es gelten außerdem $\rho(r, 0)=0$ und $\rho(r, 2 \pi)=2 \pi$. Somit besteht, wie oben überlegt wurde, die Darstellung

$$
1+\frac{z f^{\prime \prime}(z)}{f^{\prime}(z)}=\frac{1}{2 \pi} \int_{0}^{2 \pi} \frac{e^{i \varphi}+z}{e^{i \varphi}-z} d \rho(\varphi),
$$

worin $\rho(\varphi)$ eine monoton wachsende beschränkte und überdies $\rho(0)=0$ und $\rho(2 \pi)=2 \pi$ genügende Funktion bedeutet. Daher kann man sofort die Abschätzungen 


$$
\frac{1-|z|}{1+|z|} \leqq\left|1+\frac{z f^{\prime \prime}(z)}{f^{\prime}(z)}\right| \leqq \frac{1+|z|}{1-|z|}
$$

schließen, und weiter aus der der Division durch $z$ nachfolgenden Integration der soeben erhaltenen Darstellung unmittelbar folgenden Beziehung

$$
\lg f^{\prime}(z)=\frac{1}{\pi} \int_{0}^{2 \pi} \lg \frac{e^{i \varphi}}{e^{i \varphi}-z} d \rho(\varphi)
$$

gewinnt man noch die Abschätzungen

$$
\operatorname{Min}_{0 \leq \varphi \leq 2 \pi} 2\left|\lg \frac{e^{i \varphi}}{e^{i \varphi}-z}\right| \leqq\left|\lg f^{\prime}(z)\right| \leqq \operatorname{Max}_{0 \leq \varphi \leq 2 \pi} 2\left|\lg \frac{e^{i \varphi}}{e^{i \varphi}-z}\right|
$$

und beide bekannte Verzerrungsungleichungen

$$
\frac{1}{(1+|z|)^{2}} \leqq\left|f^{\prime}(z)\right| \leqq \frac{1}{(1-|z|)^{2}} \quad \text { und }\left|\arg f^{\prime}(z)\right| \leqq 2 \arcsin |z|
$$

Man bestätigt dabei leicht, daß jedes Gleichheitszeichen in diesen Verzerrungsformeln nur dann gilt, wenn die Funktion $f(z)$ eine Abbildung des Einheitskreises auf eine Halbebene vermittelt, d.h. die Gestalt $z /(1+\varepsilon z)$ mit $|\varepsilon|=1$ besitzt.

Beachtet man ferner, daß die Funktion $-2 \lg \left(1-e^{-i \varphi} z\right)$ für alle $\varphi$ jeden konzentrischen Kreis $|z| \leqq r(<1)$ auf ein und dasselbe Gebiet, also wie das der Funktion $-2 \lg (1-z)$, welches offenbar konvex ist, so folgt daraus wegen der Monotonität von $\rho(\varphi)$ mit $\rho(2 \pi)-\rho(0)=2 \pi$ der folgende von Strohhäcker herrührende Satz: Für beliebige konvex abbildende normierte schlichte Funktionen liegt jeder Punkt $f^{\prime}\left(z_{0}\right)$ $\left(\left|z_{0}\right| \leqq r<1\right)$ immer in demjenigen abgeschlossenen Gebiet. das durch die Abbildungsfunktion $z /(1-z)^{2}$ aus $|z| \leqq r$ entsteht.

Auch im Falle der Abbildungsfunktionenklasse, deren jede Funktion schlichte normierte Abbildung des Einheitskreises auf ein Sterngebiet vermittelt, kann man ganz ähnlicherweise verfahren und somit die entsprechenden Resultate herleiten, indem man dabei beachtet, daß für diese Klasse immer die Ungleichung

$$
\Re \frac{z f^{\prime}(z)}{f(z)}>0 \quad(|z|<1)
$$

gilt, und die Herglotzsche Darstellung auf die Funktion $z f^{\prime}(z) / f(z)$ anwendet.

4. Herleitung der Schwarz-Christoffelschen Formel über Polygonalabbildung. Wir sollen jetzt zeigen, daß die sog. Schwarz-Christoffelsche Transformationsformel für eine Funktion, welche den Einheitskreis in der $z$-Ebene auf das Innere eines in der $w$-Ebene gelegenen geradlinigen einfachen Polygons abbildet, auch unmittelbar aus unserer Darstellung hergeleitet werden kann. Bezeichnen wir nämlich die Eckpunkte eines Bildpolygonalgebiets mit $f\left(e^{i \varphi} \mu\right)(\mu=1, \ldots, m)$ und die inneren Winkel an diesen Punkten mit $\alpha_{\mu} \pi$, dann reduziert sich die oben angegebene Darstellung hierbei auf eine explizite Gestalt 


$$
\frac{f^{\prime \prime}(z)}{f^{\prime}(z)}=\frac{1}{\pi} \int_{0}^{2 \pi} \frac{1}{e^{i \varphi}-z} d \arg d f\left(e^{i \varphi}\right)=\sum_{\mu=1}^{m} \frac{1-\alpha_{\mu}}{e^{i \varphi_{\mu}-z}},
$$

und ergeben sich also daraus nach sukzessiven Integrationen beide Beziehungen

und

$$
\lg \frac{f^{\prime}(z)}{f^{\prime}(0)}=\sum_{\mu=1}^{m}\left(1-\alpha_{\mu}\right) \lg \frac{e^{i \varphi_{\mu}}}{e^{i \varphi_{\mu}-z}}
$$

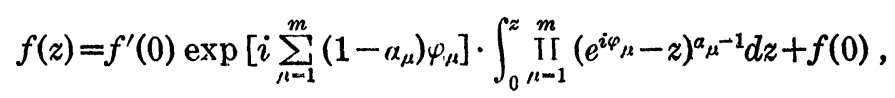

womit die gesuchte Formel erhalten ist.

5. Darstellung derjenigen Funktion, welche einen konzentrischen Kreisring auf ein Polygonalringgebiet abbildet. Falls es die entsprechende Aufgabe für zweifach zusammenhängende Gebiete betrifft und man dabei einen konzentrischen Kreisring als Grundgebiet wählt, dann kann man, von einer Villatschen Darstellungsformel über die in ihm regulär analytischen Funktionen ausgehend, in ganz analoger Weise wie oben verfahren. So ergeben sich die folgenden Resultate, deren Beweise ich in einem bald im Jap. Journ. Math. erscheinenden Bericht ausführlich angeben werde.

Eine reguläre Funktion $w=f(z)$ bilde den konzentrischen Kreisring $q<|z|<1$ auf dasjenige in der $w$-Ebene gelegene geradlinig begrenzte schlichte Polygonalringgebiet $a b$, dessen äußere Randkomponente ein Polygon mit den Eckpunkten $f\left(e^{i \varphi} \mu\right)$ von inneren Winkeln $\alpha_{\mu} \pi(\mu=1, \ldots, m)$ sei und dessen innere Randkomponente auch ein anderes Polygon mit den Eckpunkten $f\left(q e^{i \psi_{\nu}}\right)$ von inneren (bezüglich des Bildgebiets aber , äußeren “) Winkeln $\beta_{\nu} \pi(\nu=1, \ldots, n)$ sei. Dann gilt

$$
f(z)=C \int^{z} z^{i c *-1} \frac{\prod_{\mu-1}^{m} \sigma\left(i \lg z+\varphi_{\mu}\right)^{\alpha_{\mu}-1}}{\prod_{\nu=1}^{n} \sigma_{3}\left(i \lg z+\psi_{\nu}\right)^{\beta_{\nu}-1}} d z+C^{\prime}
$$

mit zwei nur von der Größe und der Lage des Bildgebiets abhängigen Konstanten $C$ und $C^{\prime}$, worin die Bezeichnungen $\sigma$ und $\sigma_{3}$ alle sich auf die der Weierstraßischen Theorie derjenigen elliptischen Funktionen beziehen, welche die Fundamentalperioden $2 \pi$ und $-i \lg q$ besitzen, und weiter die Konstante $c^{*}$ durch

$$
c^{*}=\frac{\eta_{1}}{\pi} \cdot\left\{\sum_{\mu=1}^{m}\left(1-\alpha_{\mu}\right) \varphi_{\mu}-\sum_{\nu=1}^{n}\left(1-\beta_{\nu}\right) \psi_{\nu}\right\}
$$

geliefert wird.

Ferner rẹduziert sich diese Darstellung beim Grenzübergang $q \rightarrow 0$ auf die oben angegebene Schwarz-Christoffelsche im einfach zusammenhängenden Falle. 